\title{
Impact of domain knowledge on blinded predictions of binding energies by alchemical free energy calculations
}

\author{
Antonia S. J. S. Mey ${ }^{1} \cdot$ Jordi Juárez Jiménez $^{1} \cdot$ Julien $^{\text {Michel }}{ }^{1}$
}

Received: 15 June 2017 / Accepted: 3 November 2017 / Published online: 13 November 2017

(C) The Author(s) 2017. This article is an open access publication

\begin{abstract}
The Drug Design Data Resource (D3R) consortium organises blinded challenges to address the latest advances in computational methods for ligand pose prediction, affinity ranking, and free energy calculations. Within the context of the second D3R Grand Challenge several blinded binding free energies predictions were made for two congeneric series of Farsenoid X Receptor (FXR) inhibitors with a semi-automated alchemical free energy calculation workflow featuring FESetup and SOMD software tools. Reasonable performance was observed in retrospective analyses of literature datasets. Nevertheless, blinded predictions on the full D3R datasets were poor due to difficulties encountered with the ranking of compounds that vary in their net-charge. Performance increased for predictions that were restricted to subsets of compounds carrying the same net-charge. Disclosure of X-ray crystallography derived binding modes maintained or improved the correlation with experiment in a subsequent rounds of predictions. The best performing protocols on $\mathrm{D} 3 \mathrm{R}$ set 1 and set 2 were comparable or superior to predictions made on the basis of analysis of literature structure activity relationships (SAR)s only, and comparable or slightly inferior, to the best submissions from other groups.
\end{abstract}

Keywords D3R - Computer-aided drug design · Proteinligand interactions . Alchemical free energy calculations

Electronic supplementary material The online version of this article (https://doi.org/10.1007/s10822-017-0083-9) contains supplementary material, which is available to authorized users.

Julien Michel

mail@julienmichel.net

1 EaStCHEM School of Chemistry, University of Edinburgh, David Brewster Road, Edinburgh EH9 3FJ, UK

\section{Introduction}

There is growing interest in the routine use of alchemical free energy (AFE) calculations for predictions of protein-ligand binding energies in structure-based drug discovery programs [1-7]. In particular building on pioneering work over three decades ago [8,9], some modern alchemical relative free energy calculation protocols achieve in several diverse protein binding sites sufficiently accurate predictions of binding energies [root mean square deviations (RMSD) under $1.5 \mathrm{kcal} \mathrm{mol}^{-1}$; Pearson Correlation coefficient's (R) of around 0.7 or better] to speed up hit-to-lead and lead optimisation efforts [10]. In favourable cases, AFE calculations can even reproduce subtle non-additivity of structure-activity relationships [11]. However, for a given set of protein-ligand complexes it remains difficult to anticipate the predictive power of AFE calculations. Uncertainties in binding modes [12-14] protonation/tautomeric states [15, 16], binding site water content [17-19], and choice of potential energy functions [20,21], can profoundly influence the outcome of such calculations. Accordingly, there is much interest in defining as much as possible a domain of applicability for the technology [22].

Blinded prediction competitions, whereby participants submit physical properties computed by a model in the absence of knowledge of the actual experimental data, have been instrumental in driving methodological progress in a wide range of scientific fields [23-26]. Blinded predictions reduce the impact of unconscious biases on the design of protocols, and allow evaluation of molecular modelling methods in a context closer to their intended use in drug discovery. This is advantageous for academic groups that have expertise in computational methodologies, but lack resources to carry out prospective studies. It is also 
beneficial for the field to evaluate different methodologies applied to the same dataset with identical analysis protocols.

This report focuses on the predictions submitted by our group within the context of the second Drug Design Data Resource (D3R) Grand Challenge, that ran between September 2016 and February 2017. The D3R Grand challenge 2 was the second blinded prediction challenge organised by the D3R consortium in this case looking at predicting binding poses, binding affinity ranking, and free energies for a series of 102 ligands of the Frasenoid X Receptor (FXR). This complements previous reports from our group on blinded predictions of protein-ligand poses, rankings, binding free energies [27], distribution coefficients [28], and host-guest binding free energies [29], within the frame work of the first D3R challenge in 2015 and the SAMPL5 challenges [30, 31]. The dataset of 102 inhibitors of FXR, both crystal structures and affinity data, were provided by Roche. The competition featured pose predictions, dataset rankings, and relative binding free energy predictions for two subsets of 15 and 18 compounds referred to as set 1 and set 2 respectively. Our group only submitted predictions of the relative binding free energies for the set 1 and set 2 subsets. Submissions were made before (stage1) and after (stage2) information about binding poses of representative set 1 or set 2 compounds were made available. This enabled an analysis of the impact of the available experimental data on the performance of the protocols. All input data download and submissions upload were conducted via the website of the D3R consortium [32].

\section{Theory and methods}

\section{Datasets}

\section{Blinded datasets}

At the start of the challenge (stage 1 ), the organisers released the pseudo apo-protein structure of ligand $\mathbf{1 0}$ as provided by Roche, as well as 36 ligands in SDF format to be used for the prediction of crystallographic poses, and an additional set of 66 ligands that should be used in affinity rankings. There were two subsets identified among these 102 ligands, set 1 with 15 compounds and set 2 with 18 compounds, for which relative binding free energies could be calculated. Ligand subsets set1 and set2 are depicted in Fig. SI2. For the second stage of the challenge, $36 \mathrm{X}$-ray structures were released, meaning that they could be used to prepare input files for alchemical free energy calculations. Once the competition was over a set of IC50 data for the entire dataset was released. The data stems from a scintillation proximity assay using only the FXR binding domain and a radioactive tracer. More information on the experimental binding assay as well as a study on other FXR inhibitors can be found in a series of publications [33-36]. Experimental relative binding free energies were estimated by Eq. 1

$\Delta \Delta G_{L 1 \rightarrow L 2}=k_{B} T \ln \frac{I C_{50(\mathrm{~L} 2)}}{I C_{50(\mathrm{~L} 1)}}$,

where $\mathrm{L}_{1}$ and $\mathrm{L}_{2}$ represent two ligands for which a relative energy difference is computed and $k_{B}$ and $T$ are the Boltzmann constant and temperature respectively.

\section{Literature datasets}

In order to test the computational protocols before submission of blinded predictions, retrospective studies were carried out using available literature data. A set of inhibition and structural data for 3-aryl isoxazole analogs of the nonsteroid agonist GW4064 had been previously published [34, 36]. The data consists of two different ligand series, where the first series contains eight compounds (LitSet1) and the second series 17 (LitSet2). The same experimental IC50 assay as described for the blinded dataset was used. Relative binding free energies were computed from the reported IC50s with Eq. 1. A summary of the compounds present in LitSet1 and LitSet2 can be found in Fig. SI1.

\section{Methods}

The methodology used for the calculations of relative binding free energies of FXR ligands was a single topology molecular dynamics alchemical free energy approach. Several operations are necessary to produce a set of output relative free energies of binding, based on a input set of protein antom coordinates and 2D descriptions of ligands. Currently this is implemented by a semi-automated workflow as depicted in Fig. 1.

\section{Initial protein and ligand structure setup}

For the two sets of literature data, the crystal structure with PDB ID 3FXV (FXR in complex with compound 7a) was used for the ligands taken from Feng et al. [34], and the crystal structure with PDB ID 3OKI (FXR in complex with compound 1a) was used for data taken from Richter et al. [36].

Due to the plasticity of the binding site of FXR and the differences in shape between compounds in set 1 and set2, two different protein structures were needed to build complexes between FXR and compounds of set 1 and set 2 . Each structure required a different preparation protocol. For set1 the FXR structure provided by the organizers was chosen as an initial template. For the docking calculations, that mainly consider residues delineating the binding site, the standard protein preparation workflow in Maestro 11 (beta) and conversion to the appropriate format with the utility fconv was 
Fig. 1 Semi-automated workflow for predicting relative free energies of binding. Workflow operations are depicted by blue boxes. Green boxes denote software available for automated execution of the workflow step. Red boxes denote operations that require human intervention

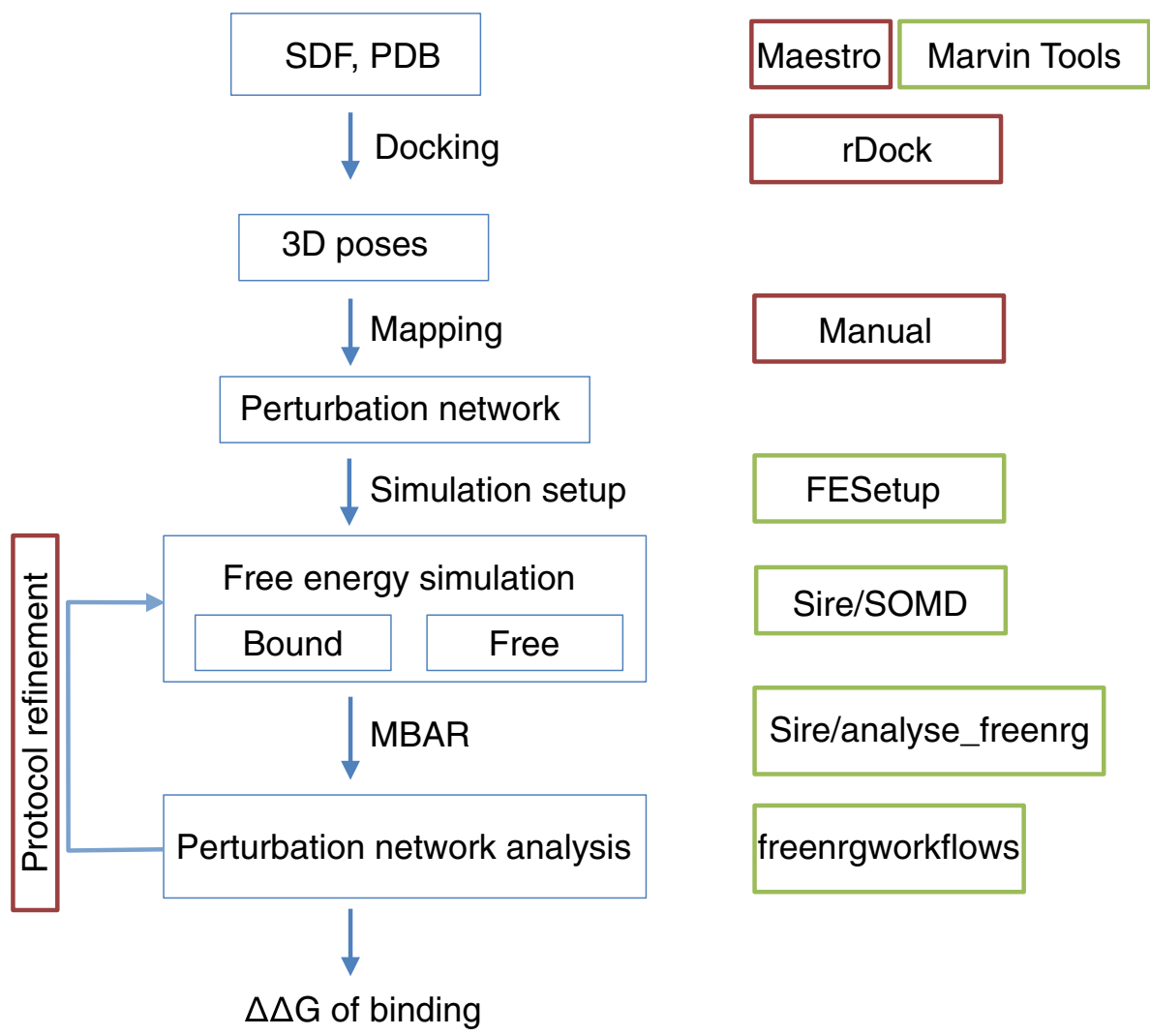

sufficient. To use the resulting structure in alchemical free energy simulations, however, it was necessary to model the missing region comprised between residues A459 and K464. Visual analysis of crystallographic structures available in the PDB revealed that fragments of the region comprised between M450 and N472 are missing in several structures (i.e: $3 \mathrm{FXV}$ ), or are arranged in at least two slightly different conformations. The first conformation displays a slightly kinked alpha helix spanning from residue N432 to residue N461 with a loop connecting residues D462 to T466 (as in structure $3 \mathrm{OKH}$ ). In the second conformation the kinked alpha helix is shorter (N432 to S457) and the loop is longer (W458 to T466) and adopts a different orientation (as in structure 3OKI). After superimposing the structure provided by the organizers with representative structures of each conformation, $3 \mathrm{OKH}$ was deemed as a suitable template to build the missing fragment of the structure. Subsequently, appropriate capping groups were added to residue M247 of the main chain and to residues D743 and D755 of the co-activator fragment. For set2, the 3OKI structure was used as an initial template and the preparation process was significantly simpler. The standard protein structure preparation workflow of Maestro 11 (beta) with addition of capping groups was sufficient to generate structures suitable for both docking and FEP calculations.

Ligand 3D structures compatible with the assay conditions were generated from 2D SDF files provided by the organizers using MarvinTools scripts available in Marvin Sketch 15.3.30 software package. The $p K a$ predictor available in the same package was used to evaluate the major protomer/tautomer for these compounds bearing ionizable substituents. No crystallographic water molecules were retained for the docking calculations.

\section{Generation of ligand binding modes}

Binding modes for the literature data were manually build in Maestro 11 (beta) by means of an overlay with the binding mode of compounds 7a and $\mathbf{1 a}$ as observed in the X-ray crystal structures $3 \mathrm{FXV}$ and $3 \mathrm{OKI}$ respectively.

For set 1 of the blind datasets, a putative binding mode for the series was obtained by docking the compounds bearing the smallest (hydrogen, 91) the bulkiest (morpholino amide, 102) substituent, as well as compound $\mathbf{1 0 1}$ to probe the effect of an ionized carboxylic acid on the binding mode. Consistent binding modes were obtained for the three molecules in the crystallographic structure provided by the organizers. To minimize the differences between binding modes within the set 1 series, the binding modes for all compounds were manually created from the binding mode of the largest compound 102. A similar protocol was followed for compounds in set2, using compounds $12,74,76,79$ and 83 to explore the influence of different substituents in the sulfonamide. A consistent binding mode was found for these compounds in 
the protein conformation displayed in PDB ID 3OKI, and putative binding modes for the entire series were manually created from the binding mode of compound $\mathbf{8 3}$.

All docking calculations were performed with rDock, generating the cavity using the two-sphere method available in the program, centering a $15 \AA$ cavity within residues M294, I356, S336 and Y373 using 1.5 and $4.0 \AA$ for the radius of the small and large spheres respectively. Manual building of the compounds was performed with Maestro 11 (beta) and the minimizer available in the suite was used to avoid steric clashes. After poses were obtained, water molecules resolved in the X-ray structure provided by the organizers were superimposed with the coordinates of the poses. Clashing water molecules were displaced to nearby positions. For the second stage of the challenge, the additional knowledge gained from the crystal structures was leveraged to prepare new input files for the alchemical free energy calculations.

\section{Alchemical calculations input preparation}

Once a set of satisfactory $3 \mathrm{D}$ poses for both set 1 and set2 was obtained, a relative free energy perturbation network was manually designed for both set 1 and set 2 ligands. The network was assigned in such a way that resulting perturbations between structures would be minimal and as many as possible simple cycles would be contained in the network in order to allow for cross validation using cycle closure as a measure. Set1 included one ambiguous binding mode for compound 47. For set 2 only three of the 18 compounds had a clearly preferred binding mode. Typically there was uncertainty in the position of ortho or meta substituents of a benzyl ring. Whenever there was ambiguity, the different binding modes were included in the perturbation map. The perturbation networks can be found in Figs. 3-7 of the SI. With the perturbation networks defined, the software FESetup [37] release $1.3 \mathrm{dev}$, was used to parametrise set 1 and set 2 ligands, setup ligands in a water box as well as protein environment and create the needed input for the alchemical free energy simulations.

Ligands Ligands were parametrised using the generalised amber force field 2 (GAFF2) [38], followed by solvation in a rectangular box of $12 \AA$ length using TIP3P water $[39,40]$. An energy minimization using a steepest decent algorithm with 500 steps was carried out on the water box, followed by an NVT simulation with the ligand restrained, during which the system was heated to $300 \mathrm{~K}$ over 1000 steps. Next an NPT equilibration at $1 \mathrm{~atm}$ was run for 5000 steps, followed by the release of the restraint on the ligand over 500 steps. FESetup used the software pmemd for this equilibration. For each perturbation a SOMD compatible perturbation file was then created from the perturbation map produced by FESetup.

Protein-ligand complex For the protein and ligand complex the protein and previously parametrised ligands were combined and solvated in a rectangular box of $10 \AA$. The protein forcefield was the amber 14 SB forcefield [38]. An equivalent solvation and equilibration protocol was used as described for the solvated ligand only.

\section{Alchemical free energy simulations}

The alchemical free energy protocol used here is based on the SOMD software as available in the Sire 2016.1.0 release [41]. This version of SOMD is linked with OpenMM 7.0.1 [42] that provides a CUDA compatible integrator enabling simulations to be run on a cluster of GPUs.

Details about the theoretical background are available elsewhere [4, 6, 7, 10, 43-46]. The main idea behind alchemical free energy calculations is to avoid direct computation of the free energy change associated with the reversible binding of a ligand to a protein. Instead one computes the free energy change for artificially morphing a ligand $\left(L_{1}\right)$ into another ligand $\left(L_{2}\right)$. By introducing a parameter $\lambda$, which defined the change from $L_{1}$ to $L_{2}$. Practically, either a replica exchange algorithm is used to simulate at different $\lambda$ windows, or a set of discrete $\lambda$ simulations is carried out. Repeating this process for $L_{1}$ and $L_{2}$ in aqueous solution or bound to the protein of interest enables construction of a thermodynamic cycle that yields the relative binding free energy of the two ligands.

Each alchemical free energy calculation for a pair of ligands $L_{1}$ and $L_{2}$ consisted minimally of one forward ( $L_{1}$ to $L_{2}$ ) and one backward $\left(L_{2}\right.$ to $\left.L_{1}\right)$ computation. Ligand pairs that showed poor agreement between forward and backwards simulation were repeated up to three times. Mean free energies and standard error were estimated from the resulting distributions of computed relative binding free energies. Further details are provided in the SI [47]. All simulations shared the following common set of parameters. Each simulation box was treated with periodic boundary conditions and simulations were run for $4 \mathrm{~ns}$ each using a 2 fs integration timestep with a Leap-Frog-Verlet integrator. Bonds involving hydrogens were constrained, except if the hydrogen atom was morphed to a heavy atom in the perturbation. The temperature was maintained at $298 \mathrm{~K}$ using an Andersen thermostat and a collision frequency of $10 \mathrm{ps}^{-1}$ with velocities initially drawn from a Maxwell-Boltzmann distribution of that temperature. Pressure was kept at $1 \mathrm{~atm}$ using the Monte Carlo Barostat implemented in OpenMM with an update frequency of $25 \mathrm{MD}$ steps. For non-bonded interactions an atom-based shifted Barker-Watts reaction field scheme was used with a cutoff of $10 \AA$ and the reaction 
field dielectric constant $\epsilon=82.0$. The number of $\lambda$ windows for each simulation varied for different perturbations and a summary, as well as complete simulation parameters can be found in the SI. All input files are available on a github repository [48].

\section{Free energy analysis and convergence}

Free energy changes were estimated both with thermodynamic integration and the multi state Bennett's acceptance ratio (MBAR) estimator, as implemented in pymbar (v 3.0.0 beta 2) [49], which was integrated into the Sire app analyse freenrg. The TI analysis served mainly two purposes; first, to ensure that the MBAR and TI free energy estimates for a particular perturbation are consistent to within approximately $0.5 \mathrm{kcal} \mathrm{mol}^{-1}$ and second, to test the convergence of the gradient $\frac{\partial U}{\partial \lambda}$ time series. Stationarity of the timeseries was assessed by means of the augmented Dicky-Fuller test. Non-stationary gradient timeseries in the pool of over 10,000 timeseries trajectories generated in this study were identified. This served as a basic test for convergence, and all non-stationary trajectories were repeated. Convergence was also assessed by checking whether binding free energies from forward and backward simulations were consistent, as well as cycle closures in the perturbation network. Simulations with poor cycle closures or poorly agreeing forward and backward transformations were repeated multiple times. The actual process of the free energy analysis for estimating cycle closure and overall affinities based on MBAR is described in the following. The first $5 \%$ of the trajectories were discarded to allow for equilibration before the MBAR analysis. Perturbation for morphing $L_{1}$ to $L_{2}$ and $L_{2}$ to $L_{1}$ were both simulated and resulting binding free energies were averaged for the forward and (reversed) backward perturbations. When available, averages were calculated across multiple independent repeats. The individually estimated free energy differences were then read into a Networkx (v 1.11) digraph [50]. The error estimated between repeated runs of backwards/forwards simulations served as the estimated error for each averaged network edge. Binding free energies relative of a ligand $L_{i}$ to a reference compound $L_{0}$ were then estimated by enumerating all possible simple paths connecting $L_{i}$ to $L_{0}$ in the network. The relative binding free energy and its uncertainty along a given path was obtained by summing relative binding free energies along each edge of the path and propagating errors. A simple path in a network is defined as the path between two vertices $v_{p}$ and $v_{q}$, with no vertex repeating along the path. Therefore a path between ligand $L_{q}$ and $L_{p}$ can be written as $P_{p, q}=\left(v_{1=p}, v_{2}, \ldots, v_{n=q}\right)$. This path is only valid if every pair of vertices has an entry in the weighted adjacency matrix $\left(w_{i j}\right)$, which in this cases holds the free energy difference of each perturbation. Therefore, the relative free energy along a single simple path with $n$ vertices, is be given by: $\Delta g_{p, q}=w_{p, 2},+\cdots+, w_{n-1, q}$. The associated error of the path can be obtained from the error matrix $\epsilon_{i j}$, which similarly to the weighted adjacency matrix will hold the error associated with each edge in the network. The error for a given simple path is therefore given by: $e_{p, q}=\sqrt{\left(\epsilon_{p, 2}^{2}+\ldots+\epsilon_{n-1, q}^{2}\right)}$. Based on the error associated with each simple path a weight of the path can be defined as $\omega_{p, q}=\sum_{\text {all paths }} 1 / e_{p, q}$. Therefore the relative free energy between ligand $L_{p}$ and $L_{q}$ can be defined as the weighted average of all paths, using $\omega_{p, q}$ as the path weight.

$\Delta \Delta G_{p q}=\sum_{\text {all paths }} \frac{\omega_{p, q} \Delta g_{p, q}}{\epsilon_{p, q}}$.

The corresponding error $E_{p, q}$ to the estimated free energy is give by:

$E_{p, q}=\sqrt{\sum_{\text {all paths }} \omega_{p, q} e_{p, q}}$

Thus paths that have smaller statistical errors contribute more than paths that show larger statistical errors.

If multiple binding modes for one compound were used in the network, they were combined into a free energy for a single compound using exponential averaging in the following way:

$\Delta \Delta G=-\left(k_{B} T\right) \ln \left[\sum_{k}^{N} \exp \left(-\Delta \Delta G\left(B M_{k}\right) / k_{B} T\right)\right]$,

where $k_{B}$ is the Boltzmann constant, $N$ the total number of binding modes, and $B M_{k}$ denotes the k-th binding mode.

\section{Charge scaling correction}

Initial analysis of literature datasets (see "Results" section) suggested that polarisation effects may play a significant role in FXR ligand binding energetics. While no polarisable forcefield was readily available to test this hypothesis, there has been some success in capturing polarisation effects in protein-ligand binding by $\mathrm{QM} / \mathrm{MM}$ reweighting of trajectories computed with a classical potential energy function [51]. Given the time constraints posed by the competition, no such methodologies were used here. Rather, an ad hoc protocol based on empirical scaling of ligand partial charges was implemented.

Thus the corrected free energies were given by:

$$
\Delta \Delta G_{\text {bind, scale }}\left(L_{1}, L_{2}\right)=\Delta \Delta G_{\text {bind }}\left(L_{1}, L_{2}\right)+\Delta \Delta G_{\text {scaled }}\left(L_{1}, L_{2}\right),
$$

where $\Delta \Delta G_{\text {scaled }}$ is given by:

$$
\begin{aligned}
\Delta \Delta G_{\text {scaled }}\left(L_{1}, L_{2}\right) & =\Delta G_{\text {scaled }}\left(L_{1}, \text { free }\right)+\Delta G_{\text {scaled }}\left(L_{2}, \text { bound }\right) \\
& -\Delta G_{\text {scaled }}\left(L_{1}, \text { bound }\right)-\Delta G_{\text {scaled }}\left(L_{2}, \text { free }\right),
\end{aligned}
$$


where the $\Delta G_{\text {scaled }}$ values are the free energy changes for scaling the partial charges of a ligand $L_{1}$ or $L_{2}$ in water, or bound to FXR. Such quantities were evaluated via MBAR analysis of trajectories for five evenly spaced $\lambda$ windows sampled for $1 \mathrm{~ns}$ each. Scaling factors ranged from 1 (no scaling) to 0.5 (50\% decrease in magnitude of partial charges).

\section{Errors analysis}

For the comparison of computed binding free energies and experimental binding free energies two measures are mainly used in the analysis, Pearson R and mean unsigned error (MUE). To obtain an error estimate for both these measures a bootstrapping approach is used in which the mean and standard error of each of the computed free energy estimates serve as the mean and standard deviation of a Gaussian distribution. For each estimate a new value from this Gaussian distribution is drawn until a new artificial distribution of computed free energies is sampled. This resampled distribution is then correlated to the experimental data. Repeating the process 10,000 times gives rise to a distribution of MUE and R, for which a mean and $1 \sigma$ confidence interval can be computed. This was the default protocol used to estimate metric errors. The organisers, however, chose a different way of estimating errors in the data sets to facilitate comparison between different submissions. This approach uses bootstrapping of the dataset, for which data points (both experimental and computed) are resampled with replacement until an artificial dataset of the same size is created. This is repeated 1000 times, leading to a distribution for Pearson R with $1 \sigma$ confidence intervals. All error bars in Fig. 6 have been generated in this fashion.

\section{Results}

\section{Literature datasets}

The robustness of the computational protocol was first tested with the two literature datasets LitSet1 and LitSet2. Supplementary Figs. 3 and 4 depict the perturbation network used for LitSet 1 and LitSet 2 respectively [47]. A summary of the results, comparing the calculated and measured binding free energies is given in Table 1. While the correlation between LitSet 1 computational and experimental data with $\mathrm{R}=0.84 \pm 0.05$ was deemed satisfactory, the mean unsigned error (MUE) at $3.0 \pm 0.2 \mathrm{kcal} \mathrm{mol}^{-1}$ was judged unexpectedly large. For the second dataset LitSet 2 the overall correlation $R=0.56 \pm 0.03$ is lower, however the MUE is significantly lower at $1.7 \pm 0.1 \mathrm{kcal} \mathrm{mol}^{-1}$.
Table 1 Summary of test dataset based on GW4064 compounds

\begin{tabular}{lllc}
\hline Dataset & Scaling factor & $\mathrm{R}$ & MUE $\left(\mathrm{kcal} \mathrm{mol}^{-1}\right)$ \\
\hline LitSet1 & 1.0 & $0.84 \pm 0.05$ & $3.0 \pm 0.2$ \\
& 0.9 & $0.83 \pm 0.04$ & $2.45 \pm 0.18$ \\
& 0.8 & $0.81 \pm 0.04$ & $2.24 \pm 0.23$ \\
& 0.7 & $0.78 \pm 0.08$ & $1.8 \pm 0.15$ \\
& 0.6 & $0.56 \pm 0.09$ & $2.2 \pm 0.2$ \\
& 0.5 & $0.51 \pm 0.1$ & $1.4 \pm 0.2$ \\
LitSet2 $^{\mathrm{a}}$ & 1.0 & $0.56 \pm 0.05$ & $1.77 \pm 0.08$ \\
& 0.9 & $0.54 \pm 0.05$ & $1.54 \pm 0.09$ \\
& 0.8 & $0.51 \pm 0.05$ & $1.46 \pm 0.08$ \\
& 0.7 & $0.44 \pm 0.06$ & $1.47 \pm 0.09$ \\
& 0.6 & $0.37 \pm 0.06$ & $1.61 \pm 1.7$ \\
& 0.5 & $0.23 \pm 0.07$ & $1.75 \pm 0.08$ \\
\hline
\end{tabular}

${ }^{\mathrm{a}}$ Charged compound $\mathbf{1 R}$ has been omitted from the analysis

Analysis of the pairwise alchemical free energy calculations in the two datasets suggested that calculated binding free energy changes for perturbations that involve substitution of a non polar group by a polar group were overly exaggerated with respect to experimental data. Also, LitSet 2 contained one negatively charged compound (1R, carboxylic acid) which was predicted to be ca. $30 \mathrm{kcal} \mathrm{mol}^{-1}$ less potent than its $-\mathrm{H}$ counterpart, whereas experimental data suggests weaker binding of the acid by ca. +2.6 kcal mol ${ }^{-1}$.

The binding site of FXR is rather apolar (see Fig. 2a), and it was hypothesized that changes in ligand polarisation upon transfer from bulk to the FXR binding site may play a significant role. This prompted the development of an ad hoc protocol in an attempt to capture polarisation effects as described in the "Methods" section via introduction of a set of charge scaling factors. The resulting correlation coefficient and MUE for scaling factors between 1 and 0.5 are also displayed in Table 1. Figure 8a in the SI displays the correlation between the computed and experimental results, and Fig. $8 \mathrm{~b}$ of the SI summarises the effect of changing the scaling corrections from 0.9 to 0.5 of the original charge. It was found that a scaling factor of 0.7 was the best tradeoff to minimize MUE whilst maintaing a reasonable Pearson correlation coefficient. The effects are more pronounced for LitSet1. The one exception is the charged compound $\mathbf{1 R}$ in LitSet2, for which reasonable agreement with experimental data required a scaling factor of 0.5 .

Given time-constraints no further efforts were devoted to the literature datasets, and subsequent blinded submissions were made for protocols without charge scaling correction, or with a charge scaling correction of 0.7 for free energy perturbations that maintain net-charge, and 0.5 if the net-charge varies in the perturbation. 
Fig. 2 a Depiction of the FXR binding site, with hydrophilic residues shown in red and hydrophobic residues shown in blue. b Compound 17 from set1, and predicted (orange sticks) versus observed (grey sticks) binding modes. c Compound $\mathbf{1 0}$ from set2, and predicted (orange sticks) versus observed (grey sticks) binding modes

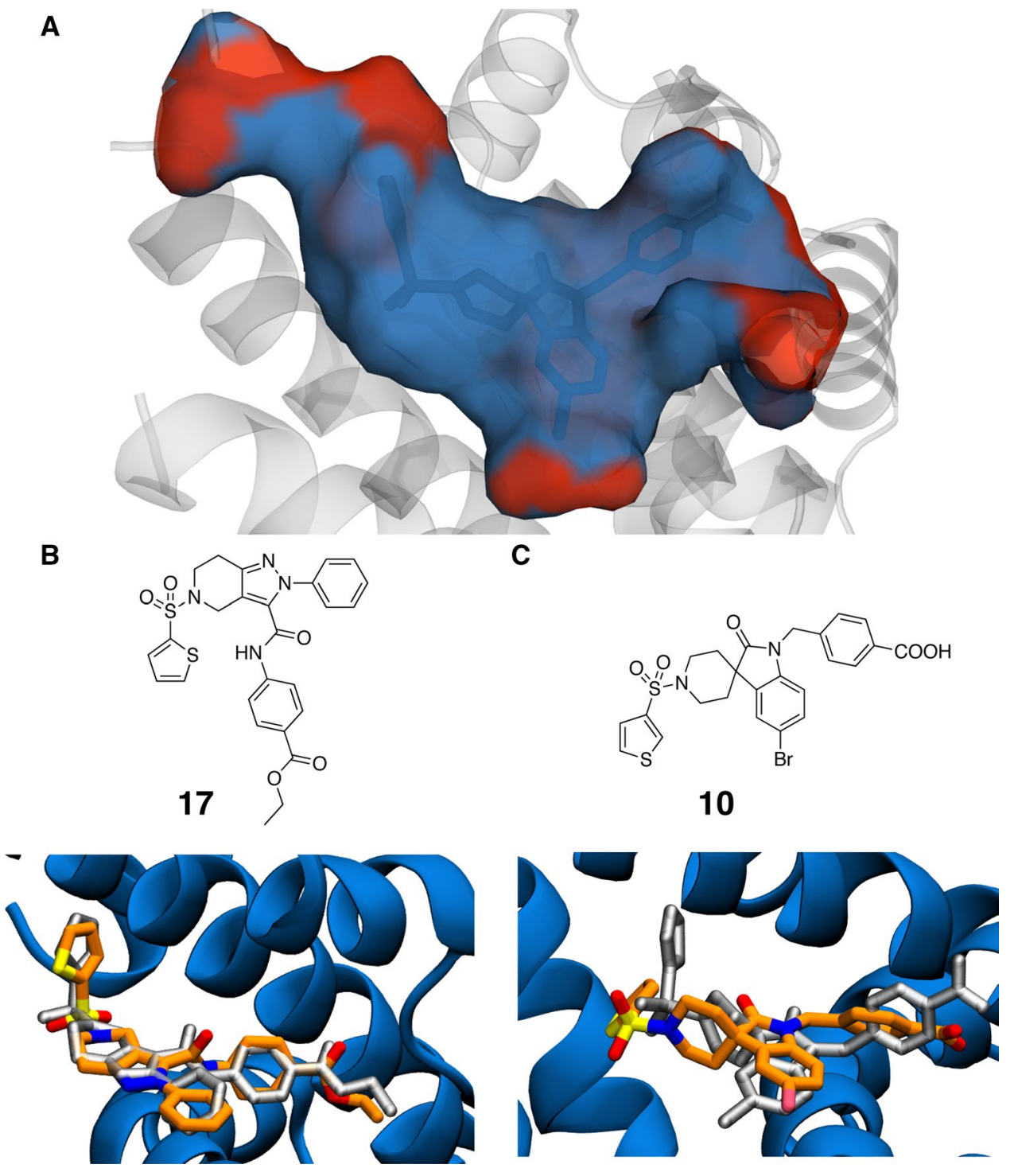

\section{Blinded dataset}

For the first stage of the competition binding modes for the FXR ligands in set 1 and set 2 had to be predicted by analysis of available crystal structures, or docking calculations as described in the "Methods" section. Figure $2 b$ top panel shows the structure of set 1 ligand $\mathbf{1 7}$, and the bottom panel depicts predicted and later disclosed binding modes. The RMSD is only $0.9 \AA$, and the binding mode prediction can be considered successful. For set2 the X-ray crystal structure of $\mathbf{1 0}$ was later disclosed. The predicted binding mode deviates more, whereas at $2.5 \AA$ the RMSD is not exceptionally high, the thiophene ring has been positioned differently to the X-ray pose. This was of concern as many of the set2 compounds feature variations in aryl sulfonamide groups.

Table 2 shows results for the protocols submitted at stage 1 of the competition. The expert opinion full protocol was a submission where binding energies were predicted by one of the authors (JM) by analysis of literature structure-activity relationships and visualisation of predicted binding modes for the LitD1 and LitD2 datasets. The expert opinion same charge protocol was not submitted but is presented to facilitate comparison with other protocols. The full protocol was a submission on the full dataset analysed as described in methods. The full guided protocol was a submission where only a small number of pathways in the perturbations network were hand-picked by one of the authors (JM) to evaluate binding free energies for the dataset. This was only done for set1. Finally the same charge protocol was a submission of alchemical free energy predictions restricted to the largest subset of compounds with the same net-charge.

For the second stage of the competition, calculations were repeated from a new set of poses for set 2 compounds. Set 1 poses were the same as in stage 1 . Additionally 
Table 2 Performance of the protocols submitted at stage 1 of the D3R competition

\begin{tabular}{lllcc}
\hline Dataset & Protocol & ID & $\mathrm{R}$ & MUE $\left(\mathrm{kcal} \mathrm{mol}^{-1}\right)$ \\
\hline Set1 & Expert opinion full & pbjwu & $0.16 \pm 0.04$ & $1.71 \pm 0.08$ \\
& Expert opinion same charge & N/A & $0.17 \pm 0.04$ & $1.76 \pm 0.09$ \\
& Full & a3c8k & $0.16 \pm 0.1$ & $2.6 \pm 0.1$ \\
& Full guided & bolbu & $0.14 \pm 0.03$ & $1.9 \pm 0.04$ \\
& Same charge & Opsyy & $0.29 \pm 0.04$ & $1.9 \pm 0.1$ \\
Set 2 & Expert opinion full & fxtpq & $0.23 \pm 0.05$ & $1.79 \pm 0.09$ \\
& Expert opinion same charge & N/A & $0.03 \pm 0.05$ & $1.72 \pm 0.1$ \\
& Full & qvnq5 & $-0.57 \pm 0.04$ & $3.18 \pm 0.22$ \\
& Same charge & f6een & $0.2 \pm 0.1$ & $1.35 \pm 0.12$ \\
\hline
\end{tabular}

Table 3 Performance of the protocols submitted at stage 2 of the D3R competition

\begin{tabular}{lllcc}
\hline Dataset & Protocol & ID & R & MUE $\left(\mathrm{kcal} \mathrm{mol}^{-1}\right)$ \\
\hline Set1 & Full & 07tpe & $0.13 \pm 0.02$ & $2.20 \pm 0.08$ \\
& Same charge & olv52 & $0.3 \pm 0.02$ & $1.41 \pm 0.08$ \\
& Full scaled & inspj & $0.12 \pm 0.02$ & $2.32 \pm 0.09$ \\
& Same charge scaled & 4botu & $0.13 \pm 0.02$ & $2.4 \pm 0.1$ \\
Set2 & Full & qt771 & $-0.44 \pm 0.02$ & $3.79 \pm 0.1$ \\
& Same charge & 0jz8u & $0.54 \pm 0.03$ & $1.67 \pm 0.08$ \\
& Full scaled & jzrt5 & $0.41 \pm 0.06$ & $1.65 \pm 0.09$ \\
& Same charge scaled & c1nbt & $0.41 \pm 0.05$ & $1.56 \pm 0.1$ \\
\hline
\end{tabular}

individual perturbations were categorised as 'easy', 'medium' or 'difficult' on the basis of the precision of the calculated relative binding free energies obtained at stage 1 , and this led to lambda schedule protocols with less, the same amount of, or more, windows as in stage 1 (see SI for details). The time left in the competition was used to carry out multiple repeats of the perturbations that showed higher statistical errors. Additionally, the optimisation of charge scaling factors on the literature datasets had been completed by then, and scaling factor corrections were also applied to set 1 and set 2 datasets. Table 3 shows the results for protocols submitted at stage 2 of the competition. Only full and same charge protocols, and their scaled variants, were submitted.

At stage 1 the expert opinion protocol shows $\mathrm{R}$ values for both set 1 and set2 of ca. 0.2, and MUE values ca. $1.7 \mathrm{kcal} \mathrm{mol}^{-1}$. The performance is similar or worse for the expert opinion same charge protocol. Alchemical free energy based protocols on the full dataset fare poorly with similar or lower R values, and higher MUE values. Submissions that only considered compounds with the same net charge show better performance ( $\mathrm{R}$ ca. 0.2-0.3, MUE ca. 1.4-1.9 kcal mol ${ }^{-1}$ ). Overall none of the protocols show satisfactory correlation with experiment.

At stage 2 of the competition, the full and same charge submissions show lower statistical errors because the additional repeats calculations on the noisier perturbations have improved convergence. For set1 the MUE decreases, but the
$\mathrm{R}$ metric is no different from stage 1 submissions. The scaled submissions for the full dataset and the same charge dataset achieve similar R values, but the MUE has worsened. For set2 lower statistical errors are also observed with respect to stage 1. The full submission produces similarly low $\mathrm{R}$ values and high MUE values. However the full scaled submission significantly increases $\mathrm{R}$ from ca. -0.4 to +0.4 , while decreasing MUE from ca. 3.8 to $1.6 \mathrm{kcal} \mathrm{mol}^{-1}$. The same charge submission shows a significant increase in $\mathrm{R}$ with respect to stage 1 (from 0.2 to ca. 0.5 ), but the MUE increases from 1.3 to $1.7 \mathrm{kcal} \mathrm{mol}^{-1}$. Finally, the same charge scaled protocol achieves a poorer R value (ca. 0.4) and similar MUE value.

Overall the most significant improvement at stage 2 is observed for the set 2 same charge dataset. This could be because the predicted binding modes at stage 1 were not in agreement with the subsequently disclosed X-ray structures. The scaling protocol appears to yield large improvements on set 2 , but this actually comes at the expense of decreased predictive power for the subset of compounds that carry the same net-charge (see below).

Figure 3 depicts detailed results for the full dataset of set1 compounds at different stages of the competition. Figure $3 \mathrm{a}$ shows at stage 1 the relative binding free energy of charged compound 101 is significantly overestimated with respect to all other neutral compounds. Compound $\mathbf{4 5}$ is also a significant outlier. Figure $3 \mathrm{~b}$ shows that at stage 2 there is a trend towards better agreement with experiment, apart from $\mathbf{1 0 1}$ and $\mathbf{4 5}$ that remain significantly off. Figure $3 \mathrm{c}$ shows that 


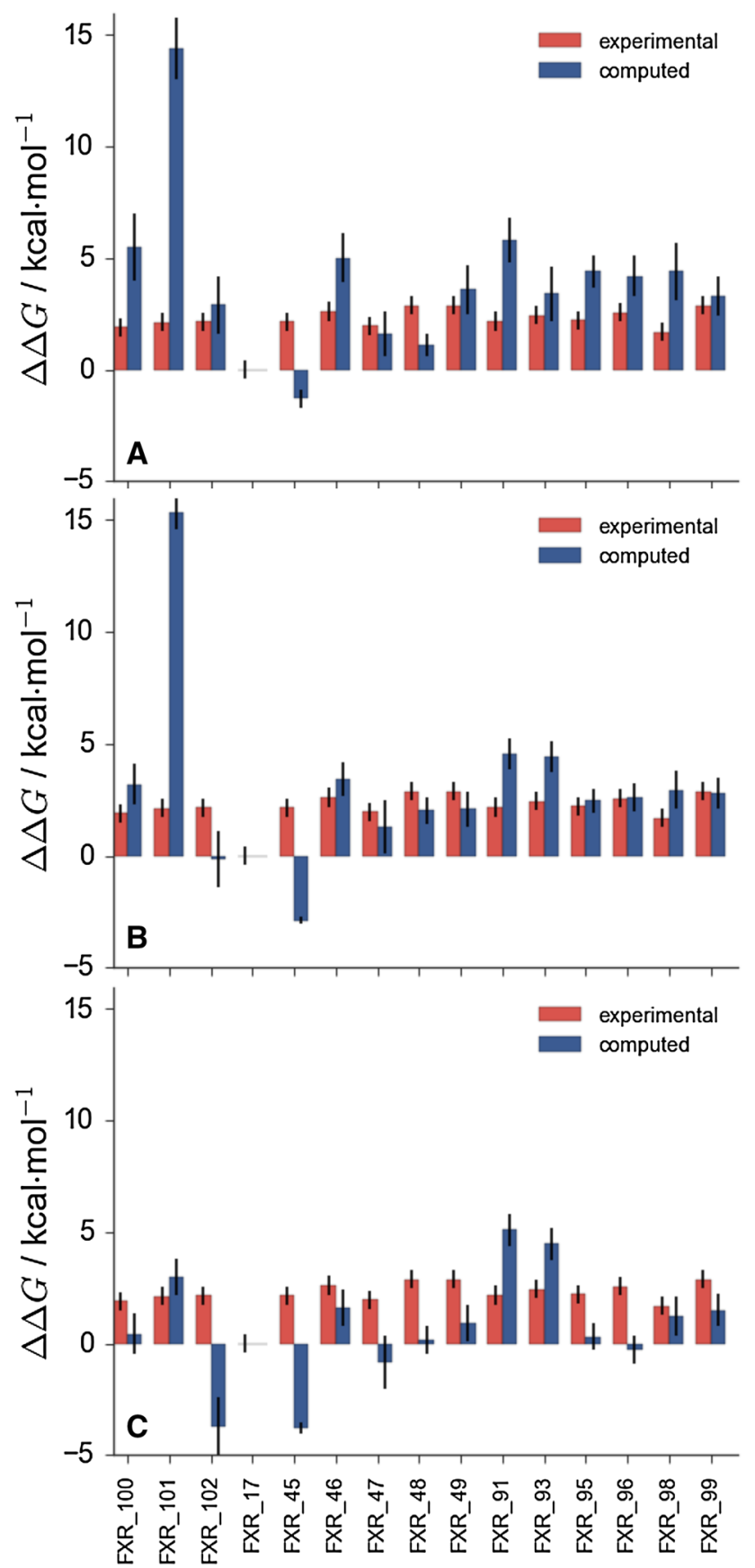

Fig. 3 a Stage1 full submission for set1(ID a3c8k) showing clear overestimation of the relative binding free energy of charged compound 101. b Stage 2 full submission (ID 07tpe). c Stage 2 full scaled submission (ID inspj)

the full scaled submission considerably improves free energy estimates for compound 101, but also drastically decreases the accuracy of estimates for neutral compounds 102, 48, 95, 96. It is not clear why predictions for 45 consistently perform so poorly.

Some highlights for the binding free energy estimations of set 2 are shown in Fig. 4. The stage 1 full submission

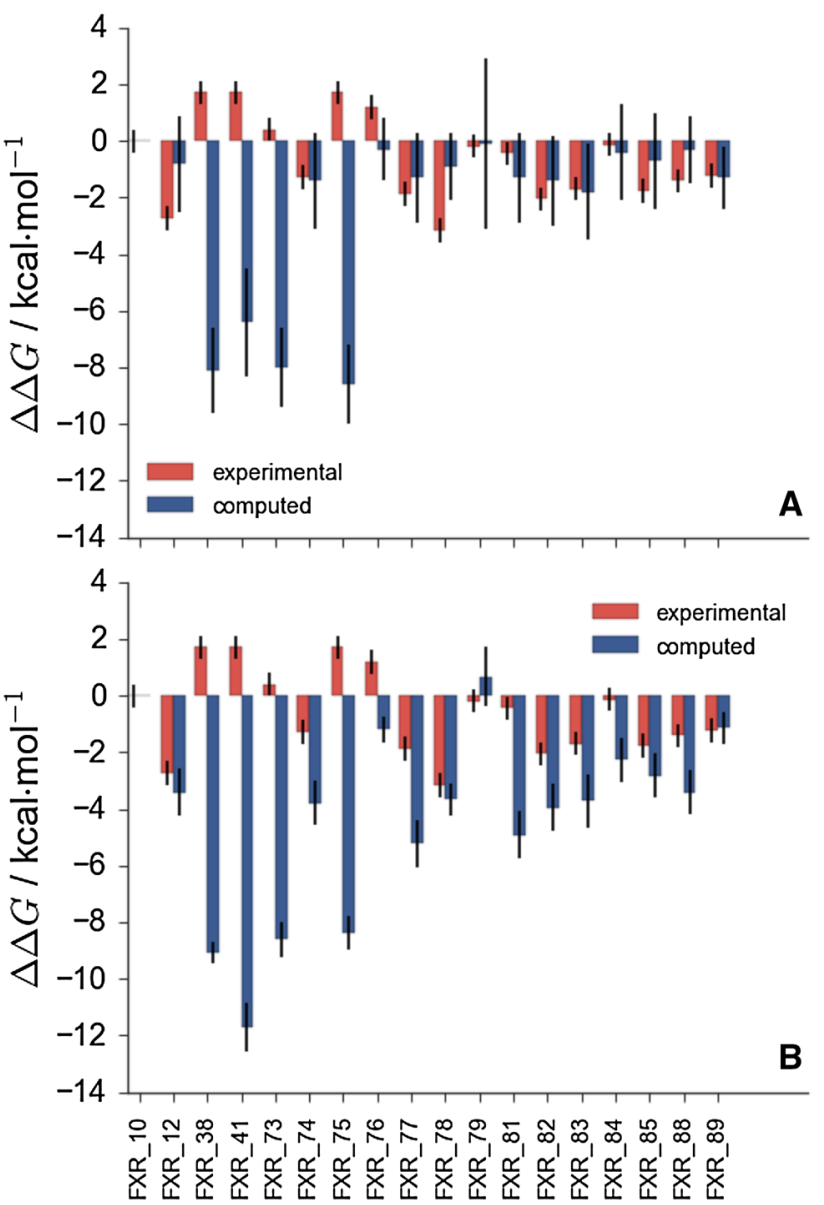

Fig. 4 a Stage 1 full submission for set2 compounds (ID qvnq5). b Stage 2 full submission for set 2 compounds (ID qt771)

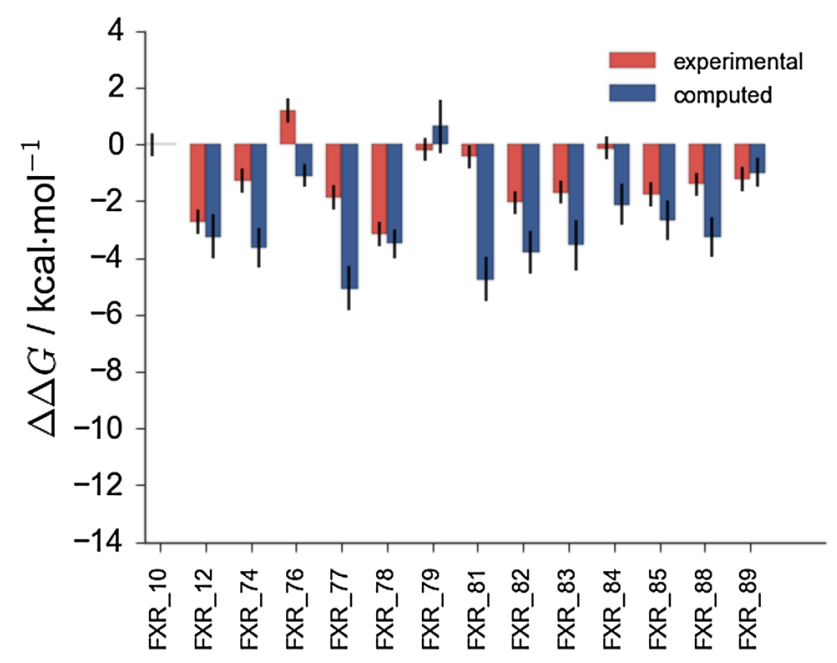

Fig. 5 Stage 2 same charge submission (ID 0jz8u) 
is depicted in Fig. 4a. The poor estimation of the relative binding free energy of neutral compounds $(\mathbf{3 8}, \mathbf{4 1}, \mathbf{7 3}, \mathbf{7 5})$ with respect to other compounds in the dataset that carry one negative charge is very apparent. Figure $4 b$ shows the full submission at stage 2 of the competition. Estimates for the charged compounds improve, however the binding free energies of the neutral compounds are still poorly estimated. This is more apparent by inspection of Fig. 5, which shows the same charge submission where only charged compounds were considered. This is the best performing protocol overall in terms of correlation coefficient of $R=0.54 \pm 0.03$ and MUE $=1.67 \pm 0.08 \mathrm{kcal} \mathrm{mol}^{-1}$.

\section{Comparison to other submissions}

The organisers released data for all binding free energy prediction submissions shortly after the end of stage 2, and a summary of the correlation coefficients can be seen in Fig. 6.
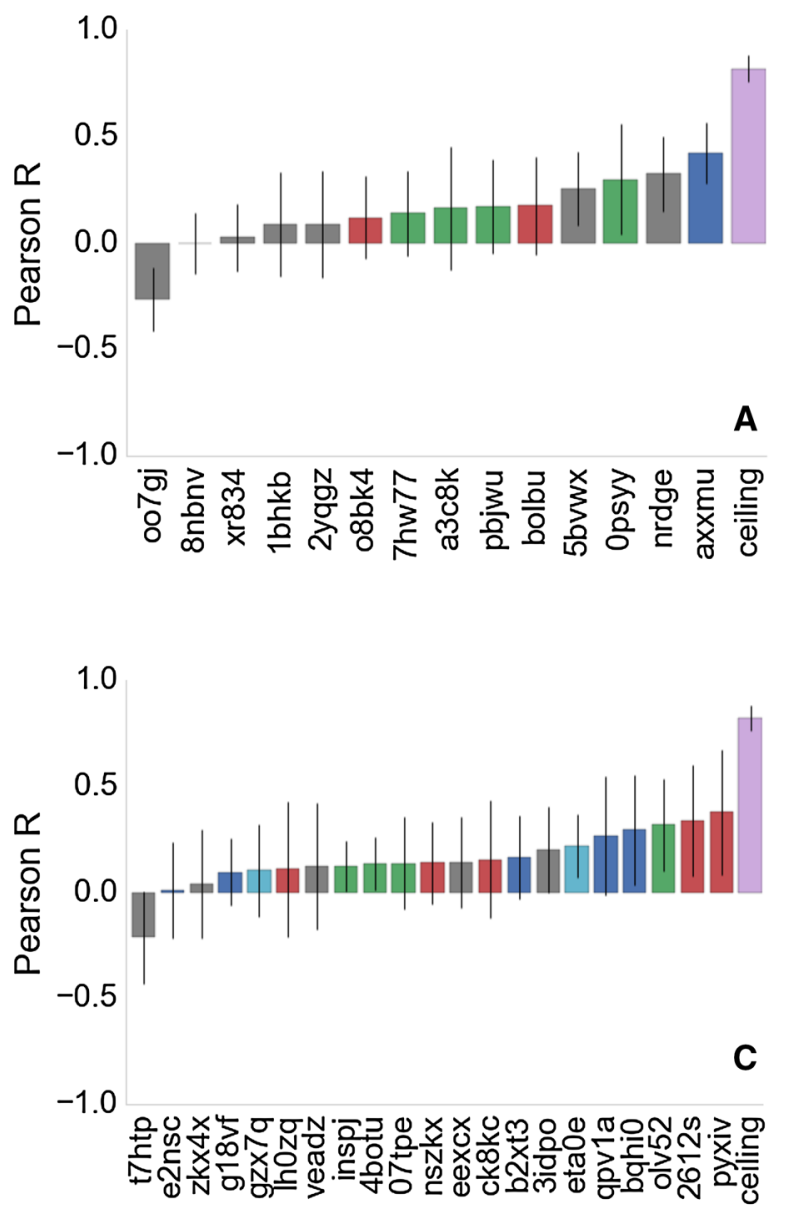

Fig. 6 Summary of all submitted protocols. a Stage 1 for set1. b Stage 1 for set2. c Stage 2 for set1. d Stage 2 for set2. Green colours denote the authors submissions and protocol IDs can be identified in Tables 2 and 3. Red colours denote other alchemical methods, blue colours denote MMPBSA based methods, the light blue colour
Figure $6 \mathrm{a}$ and $\mathrm{b}$ are stage 1 submissions for set 1 and set 2 respectively. Results for stage 2 set 1 and set 2 are shown in Fig. $6 \mathrm{c}$ and $\mathrm{d}$. The authors submissions are shown in green and can also be identified by their submission ID listed in Tables 2 and 3. It should be noted that the shown correlation coefficients are slightly different to the ones reported in the Tables 2 and 3. This is down to the use of different error analysis methods between the authors and the organisers, as discussed in the "Methods" section. However, what becomes apparent from Fig. 6 is that for set1 both at stage 1 and stage 2 there is no protocol that obviously outperforms another protocol and no statistically significant ranking is possible. For set 2 and particularly stage 2 there are four protocols that perform better than the rest, which are a mix of alchemical free energy and other protocols. Submission $81 n 55$ is an alchemical method based on FEP+, submission xk67c uses a non-equilibrium pulling approach using Gromacs as the simulation framework, submission 67a3e
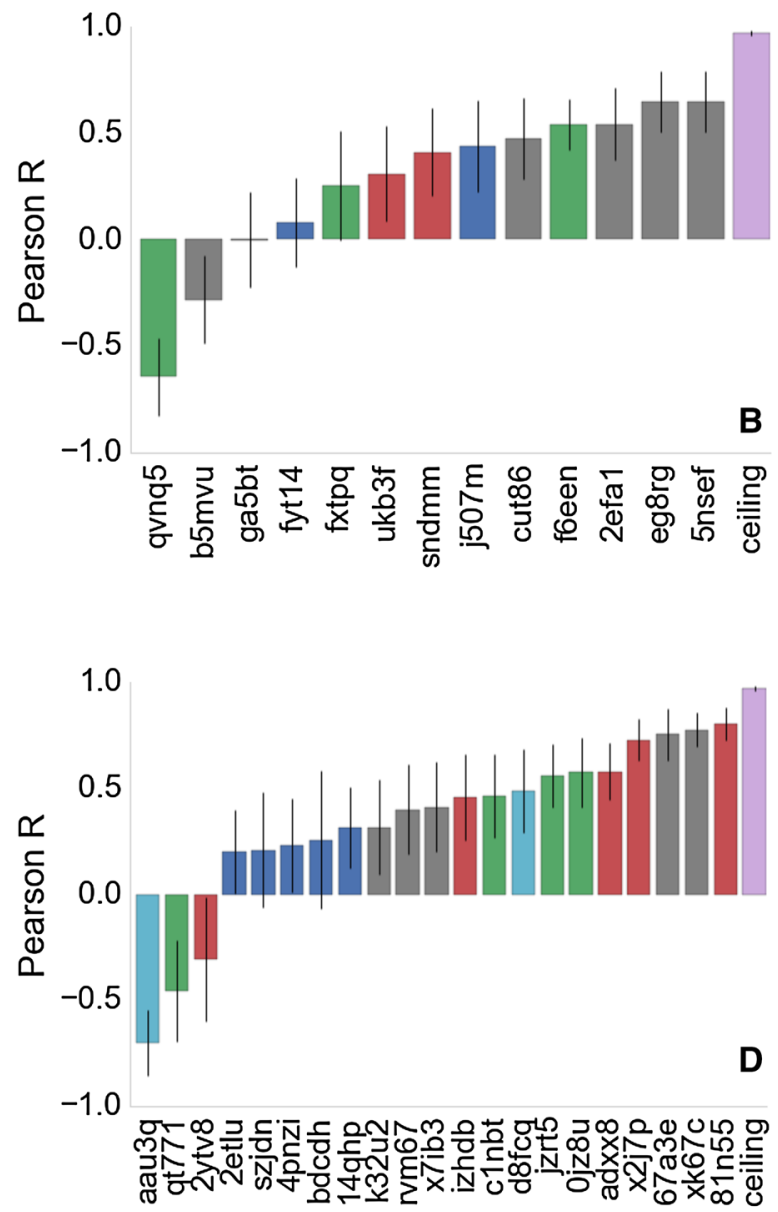

denote quantum mechanical based methods and grey denotes any other methods. The ceiling entry is discussed in the text and shown in purple. All method descriptions are made available by the organisers and can be found on the www.drugdesigndata.org website 
uses the software MIX to perform energy minimisation of protein-ligand complexes, and submission $\mathbf{x} \mathbf{2 j} \mathbf{j} \mathbf{p}$ also used FEP+.

Figure 6 suggests, that there is no clear trend indicating a method that consistently outperforms others. Furthermore, the overall correlation between predicted free energies and experimental values is poor and unreliable for set 1 .

\section{Conclusions}

The blinded predictions on FXR ligands highlighted difficulties in reliably estimating relative binding energies between compounds that differ in their net-charge with the current workflow. This was anticipated in light of past experience and until methodological advances lift this limitation, alternative ad-hoc protocols may prove more reliable. For instance, other groups submitted in this competition alchemical binding energy predictions for ligands modelled as protonated acids in order to maintain the same net-charge across the full D3R datasets. While this is an unlikely chemical state for the unbound or bound ligands given the assay conditions, this setup did lead to superior predictions for the full D3R datasets.

The relatively reasonable correlations obtained retrospectively on LitSet1 and LitSet2 ligand series were encouraging, but the high mean-unsigned error on the LitSet1 dataset prompted the development of an approximate charge scaling protocol to account for potential neglect of polarisation effects. This had no beneficial effect on the accuracy of the blinded predictions and this protocol is not recommended for further use. Overall this indicates difficulties in reliably anticipating the robustness and transferability of the protocol across different ligand series, let alone different binding sites. In spite of the difficulties encountered it is useful to note that expert opinion based on analysis of literature SARs proved no more predictive on set 1 and worse on set2 (excluding charged compounds). While this observation lacks statistical relevance-presumably there would also be variability in different expert opinions-it does highlight the difficulty of the problem. By contrast, expert opinion often fares well for poses prediction when compared against automated software workflows [27, 52]. It was also encouraging that the correlation for set 2 same-charge subset increased once experimental data about the binding mode of a representative set 2 ligand could be taken into account. By contrast no significant variation was observed for set 1 upon repeating the calculations, presumably because the binding modes had been well predicted at stage 1 of the competition.

The D3R Grand Challenge 2016 free energy datasets were markedly larger than those used in the 2015 competition. This enabled a more reliable comparison of the performance of different methodologies. Nevertheless, it is apparent that both set 1 and set 2 are still too small to reliably rank a large number of submissions made by different groups. A general trend for alchemical free energy protocols can be observed, establishing them typically in the top $33 \%$ of submission, in particular in set 2 , for both correlation coefficient and root mean square error (RMSE), as shown in the SI. It is noteworthy that the features of the distribution of experimental binding energies for set 1 (shorter span and uneven density) contribute to making predictions intrinsically more difficult than for set 2 . In addition, the precision of the experimental data was not determined. Assuming a ca. $0.4 \mathrm{kcal} \mathrm{mol}^{-1}$ uncertainty in experimental measurements [53] together with bootstrapping suggests ceiling values for $\mathrm{R}$ of ca. $0.82 \pm 0.06$ and $0.97 \pm 0.01$ for set 1 and set2 respectively. Thus the best performing methods are far from achieving high accuracy $\mathrm{R}$ values on set1, but show respectable correlation on set 2 . While it may be difficult to source significantly larger datasets amenable to alchemical free energy calculations, it may be useful to assess their intrinsic difficulty for the design of future competitions.

Acknowledgements J.M. is supported by a Royal Society University Research Fellowship. The research leading to these results has received funding from the European Research Council under the European Unions Seventh Framework Programme (FP7/2007-2013)/ERC Grant Agreement No. 336289. J. J.-J. is supported by a Marie SklodowskaCurie fellowship rant Agreement No. 655677.

Open Access This article is distributed under the terms of the Creative Commons Attribution 4.0 International License (http://creativecommons.org/licenses/by/4.0/), which permits unrestricted use, distribution, and reproduction in any medium, provided you give appropriate credit to the original author(s) and the source, provide a link to the Creative Commons license, and indicate if changes were made.

\section{References}

1. Deng Y, Roux B (2006) J Chem Theor Comput 2(5):1255-1273

2. Chang C-E, Gilson MK (2004) J Am Chem Soc 126(40):13156-13164

3. Michel J (2014) Phys Chem Chem Phys 16:4465-4477

4. Chodera JD, Mobley DL, Shirts MR, Dixon RW, Branson K, Pande VS (2011) Curr Opin Struct Biol 21(2):150-160

5. Jiang W, Roux B (2010) J Chem Theor Comput 6(9):2559-2565

6. Michel J, Foloppe N, Essex JW (2010) Mol Inform 29(8-9):570-578

7. Michel J, Essex JW (2010) J Comput Aided Mol Des 24(8):639-658

8. Jorgensen WL, Ravimohan C (1985) J Chem Phys 83(6):3050-3054

9. Tembre BL, Mc Cammon JA (1984) Comput Chem 8(4):281-283

10. Wang L, Wu Y, Deng Y, Kim B, Pierce L, Krilov G, Lupyan D, Robinson S, Dahlgren MK, Greenwood J, Romero DL, Masse C, Knight JL, Steinbrecher T, Beuming T, Damm W, Harder E, Sherman W, Brewer M, Wester R, Murcko M, Frye L, Farid R, Lin T, Mobley DL, Jorgensen WL, Berne BJ, Friesner RA, Abel R (2015) J Am Chem Soc 137(7):2695-2703 
11. Calabr G, Woods CJ, Powlesland F, Mey ASJS, Mulholland AJ, Michel J (2016) J Phys Chem B 120(24):5340-5350

12. Lim NM, Wang L, Abel R, Mobley DL (2016) J Chem Theor Comput 12(9):4620-4631

13. Michel J, Verdonk ML, Essex JW (2007) J Chem Theor Comput 3(5):1645-1655

14. Michel J, Essex JW (2008) J Med Chem 51(21):6654-6664

15. Yuan H, Sherborne B, Lee T-S, Case DA, York DM, Guo Z (2016) J Comput Aided Mol Des 30(7):533-539

16. Evoli S, Mobley DL, Guzzi R, Rizzuti B (2016) Phys Chem Chem Phys 18:32358-32368

17. Luccarelli J, Michel J, Tirado-Rives J, Jorgensen WL (2010) J Chem Theor Comput 6(12):3850-3856

18. Michel J, Tirado-Rives J, Jorgensen WL (2009) J Phys Chem B 113(40):13337-13346

19. Michel J, Tirado-Rives J, Jorgensen WL (2009) J Am Chem Soc 131(42):15403-15411

20. Mishra SK, Calabr G, Loeffler HH, Michel J, Koa J (2015) J Chem Theor Comput 11(7):3333-3345

21. Michel J, Verdonk ML, Essex JW (2006) J Med Chem 49(25):7427-7439

22. Sherborne B, Shanmugasundaram V, Cheng AC, Christ CD, DesJarlais RL, Duca JS, Lewis RA, Loughney DA, Manas ES, McGaughey GB, Peishoff CE, van Vlijmen H (2016) J Comput Aided Mol Des 30(12):1139-1141

23. Kryshtafovych A, Fidelis K, Moult J (2014) Proteins 82:164-174

24. Wodak SJ, Mndez R (2004) Curr Opin Struct Biol 14(2):242-249

25. Yin J, Henriksen NM, Slochower DR, Shirts MR, Chiu MW, Mobley DL, Gilson MK (2017) J Comput Aided Mol Des 31(1):1-19

26. Smith RD, Dunbar JB, Ung PM-U, Esposito EX, Yang C-Y, Wang S, Carlson HA (2011) J Chem Inform Model 51(9):2115-2131

27. Mey ASJS, Jurez-Jimnez J, Hennessy A, Michel J (2016) Bioorg Med Chem 24(20):4890-4899

28. Bosisio St, Mey ASJS, Michel J (2016) J Comput Aided Mol Des 30(11):1101-1114

29. Bosisio S, Mey ASJS, Michel J (2017) J Comput Aided Mol Des 31(1):61-70

30. Gathiaka S, Liu S, Chiu M, Yang H, Stuckey JA, Kang YN, Delproposto J, Kubish G, Dunbar JB, Carlson HA, Burley SK, Walters WP, Amaro RE, Feher VA, Gilson MK (2016) J Comput Aided Mol Des 30(9):651-668

31. Shirts MR, Klein C, Swails JM, Yin J, Gilson MK, Mobley DL, Case DA, Zhong ED (2017) J Comput Aided Mol Des 31(1):147-161

32. Gilson MK, Burley SK, Drug Design Data Resource, Amaro R, Feher (n.d.) An open resource to advance computer-aided drug design

33. Nichols JS, Parks DJ, Consler TG, Blanchard SG (1998) Anal Biochem 257(2):112-119

34. Feng S, Yang M, Zhang Z, Wang Z, Hong D, Richter H, Benson GM, Bleicher K, Grether U, Martin RE, Plancher J-M, Kuhn B, Rudolph MG, Chen L (2009) Bioorg Med Chem 19(9):2595-2598
35. Richter HGF, Benson GM, Blum D, Chaput E, Feng S, Gardes C, Grether U, Hartman P, Kuhn B, Martin RE, Plancher J-M, Rudolph MG, Schuler F, Taylor S, Bleicher KH (2011) Bioorg Med Chem Lett 21(1):191-194

36. Richter HGF, Benson GM, Bleicher KH, Blum D, Chaput E, Clemann N, Feng S, Gardes C, Grether U, Hartman P, Kuhn B, Martin RE, Plancher J-M, Rudolph MG, Schuler F, Taylor S (2011) Bioorg Med Chem Lett 21(4):1134-1140

37. Loeffler HH, Michel J, Woods C (2015) J Chem Inf Comput Sci 55(12):2485-2490

38. Case DA, Darden TA, Cheatham, TE, Simmerling CL, Wang J, Duke RE, Luo R, Walker RC, Zhang W, Merz KM, Roberts B, Hayik S, Roitberg A, Seabra G, Swails J, Goetz AW, Kolossváry I, Wong KF, Paesani F, Vanicek J, Wolf RM, Liu J, Wu X, Brozell SR, Steinbrecher T, Gohlke H, Cai Q, Ye X, Wang J, Hsieh MJ, Cui G, Roe DR, Mathews DH, Seetin MG, Salomon-Ferrer R, Sagui C, Babin V, Luchko T, Gusarov S, Kovalenko A, Kollman PA (2014) AMBER 12, University of California, San Francisco

39. Jorgensen WL, Chandrasekhar J, Madura JD, Impey RW, Klein ML (1983) J Chem Phys 79(2):926-935

40. Neria E, Fischer S, Karplus M (1996) J Chem Phys 105(5):1902-1921

41. Woods C, Mey ASJS, Calabro G, Bosisio S, Michel J (2016) Sire molecular simulations framework. http://siremol.org. Accessed 31 May

42. Eastman P, Friedrichs MS, Chodera JD, Radmer RJ, Bruns CM, Ku JP, Beauchamp KA, Lane TJ, Wang L-P, Shukla D, Tye T, Houston M, Stich T, Klein C, Shirts MR, Pande Vijay S (2013) J Chem Theory Comput 9(1):461-469

43. Mobley DL, Klimovich PV (2012) J Chem Phys 137(23):230901

44. Zeevaart JG, Wang L, Thakur VV, Leung CS, Tirado-Rives J, Bailey CM, Domaoal RA, Anderson KS, Jorgensen WL (2008) J Am Chem Soc 130(29):9492-9499

45. Steinbrecher T, Hrenn A, Dormann KL, Merfort I, Labahn A (2008) Bioorg Med Chem 16(5):2385-2390

46. Wang J, Deng Y, Roux B (2006) Biophys J 91(8):2798-2814

47. Mey ASJS, Juarez-Jimenez J, Michel J Supplementary information

48. Mey ASJS, Michel J (2017) Github page for simulation data. https://github.com/michellab/D3R_GC2_Data. Accessed 7 Nov 2017

49. Shirts MR, Chodera JD (2008) J Chem Phys 129(12):124105

50. Hagberg A, Swart P, Chult D (2008) Exploring network structure, dynamics, and function using NetworkX. In: Proceedings of the 7th Python in science conference (SciPy2008), Pasadena, pp $11-15$

51. Beierlein FR, Michel J, Essex JW (2011) J Phys Chem B 115(17):4911-4926

52. Reynolds CH, Merz KM Jr, Ringe D (eds) (2010) Drug design structure- and ligand-based approach, chap 7. Cambridge University Press, Cambridge

53. Brown SP, Muchmore SW, Hajduk PJ (2009) Drug Discov Today 14(7):420-427 